Supplementary Information

\title{
High Photoresponsivity of Vertical Graphene Nanosheets/p-Si Enhanced by Electron Trapping at Edge Quantum Wells
}

\author{
Siyan Gao, Liang Liu, Zezhou Lin, Xi Zhang", Dongfeng Diao \\ Institute of Nanosurface Science and Engineering, Guangdong Provincial Key Laboratory of \\ Micro/Nano Optomechatronics Engineering, Shenzhen University, Shenzhen, 518060, China \\ *Corresponding author. E-mail: zh0005xi@szu.edu.cn
}


(a)
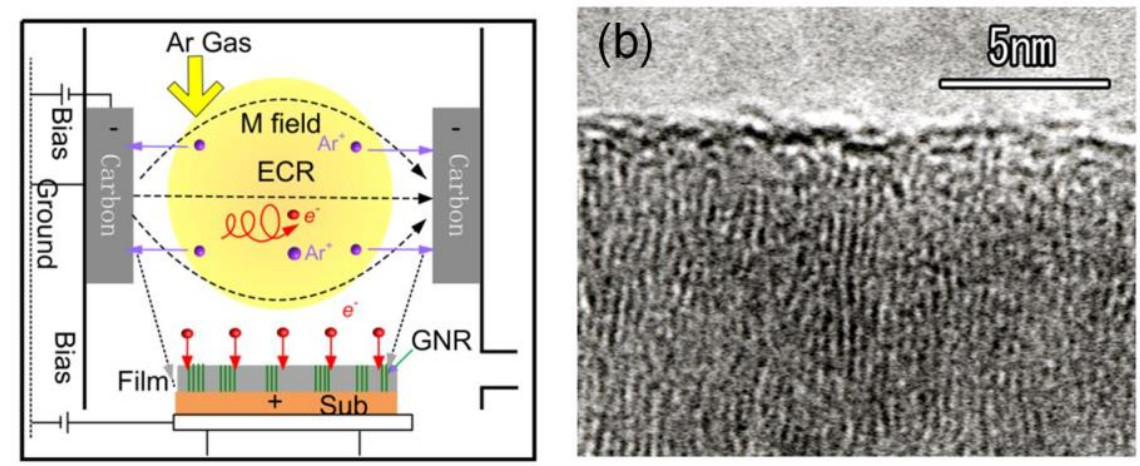

(c)

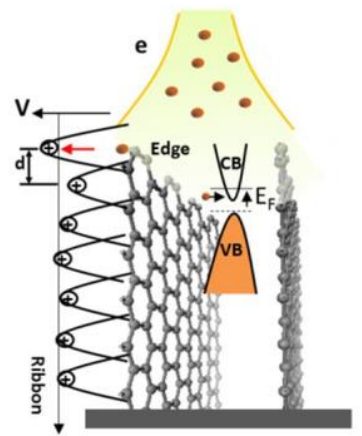

(d)

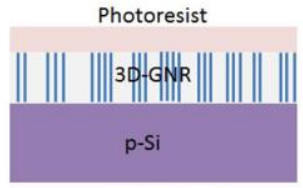

Au Sputter

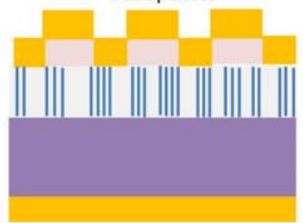

Expose to UV

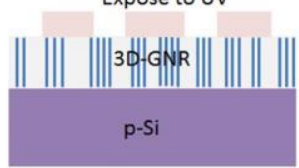

Remove

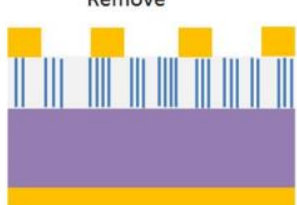

Figure S1. (a) ECR low energy electron growth of 3D-GNR film. The mirror confinement magnetic field generates high density Ar gas plasma in the chamber. A sputtering bias voltage of $-300 \mathrm{~V}$ was applied onto carbon target to generate carbon atoms for film growth. Meanwhile, electron irradiation was introduced to grow the $\mathrm{sp}^{2}$ bonding GNR. (b) TEM image of the side-view of 3D-GNR film. (c) Illustration of the growing process of 3D-GNR. (d) Photolithography of Au electrodes on 3D-GNR/p-Si photodetector. The $60 \mu \mathrm{m}$-width and 50nm-thick cross-linking gold lines are photolithographed on the top of film as negative electrode and gold at the bottom of p-silicon is positive electrode. 

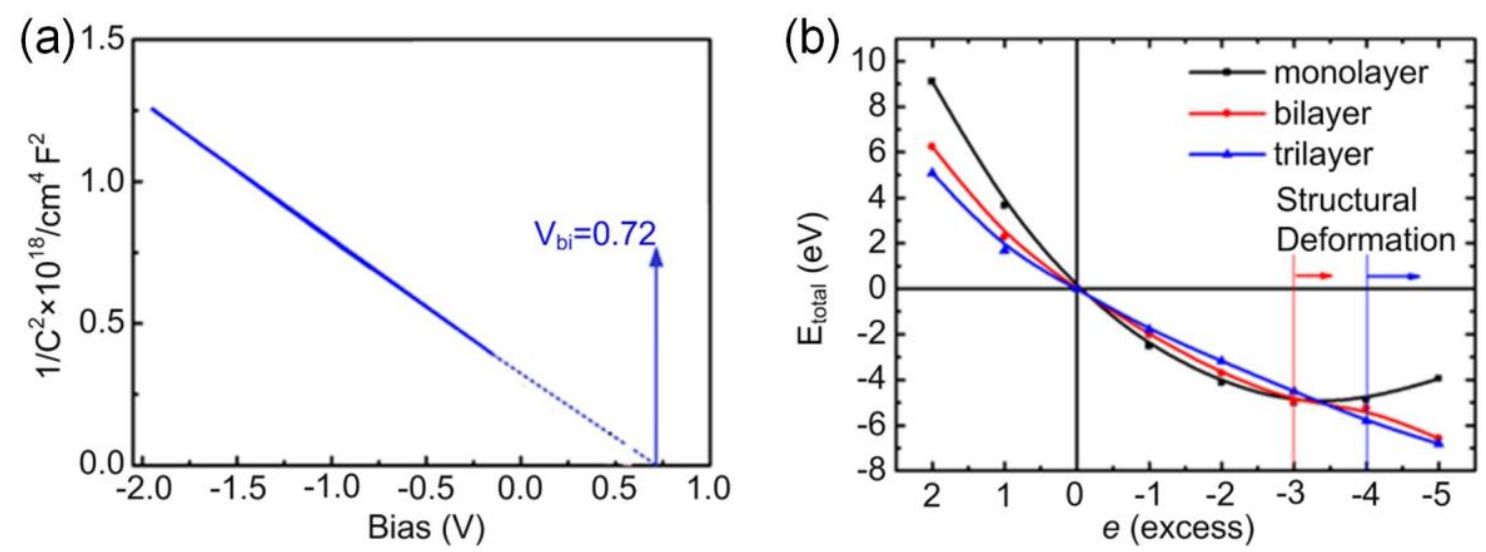

Figure S2. (a) Measurement of the inverse square of the capacitance $\left(1 / \mathrm{C}^{2}\right)$ versus bias $(\mathrm{V})$ of $3 \mathrm{D}-\mathrm{GNR} / \mathrm{p}-\mathrm{Si}$ at room temperature to derive the $\mathrm{V}_{\mathrm{bi}}=0.72 \mathrm{~V}$ and confirm the n-type behavior of 3D-GNR. (b) DFT-derived total energy versus excess electrons of monolayer, bilayer and trilayer armchair GNR. GNRs are stable with certain amount of excess electrons and keep them at edges ${ }^{1}$. Reprint from Ref. ${ }^{1}$ 
(a)

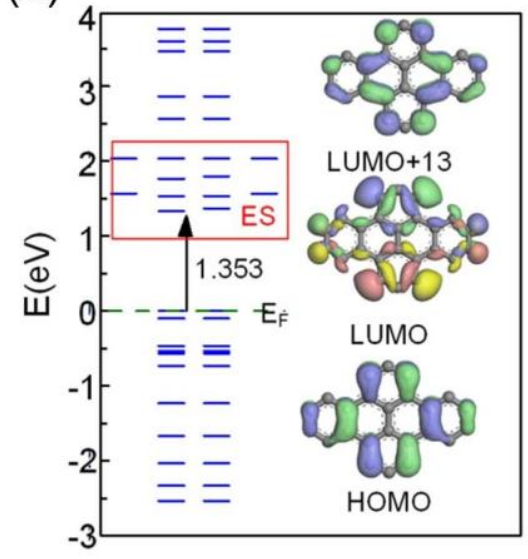

(d)

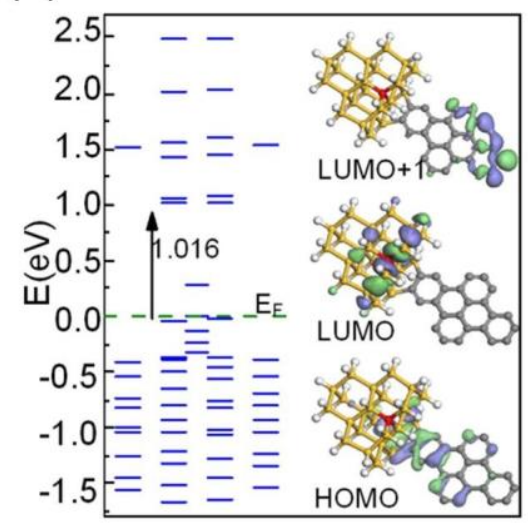

(b)

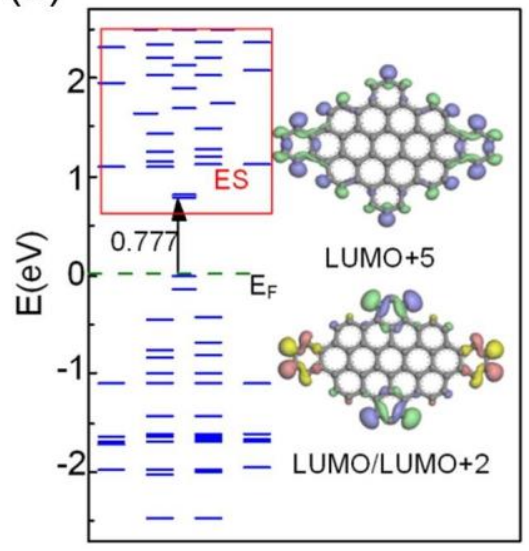

(e)

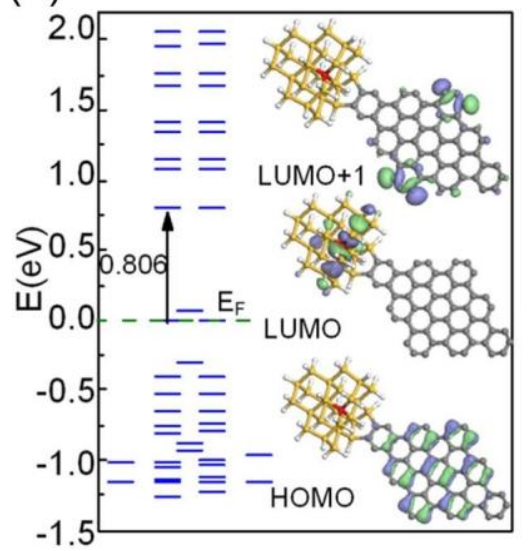

(c)

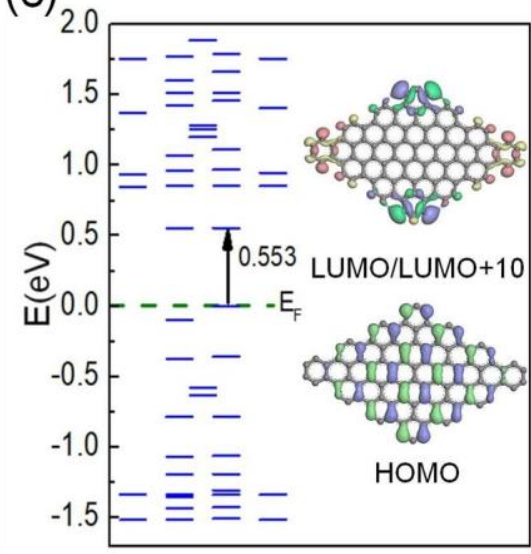

(f)

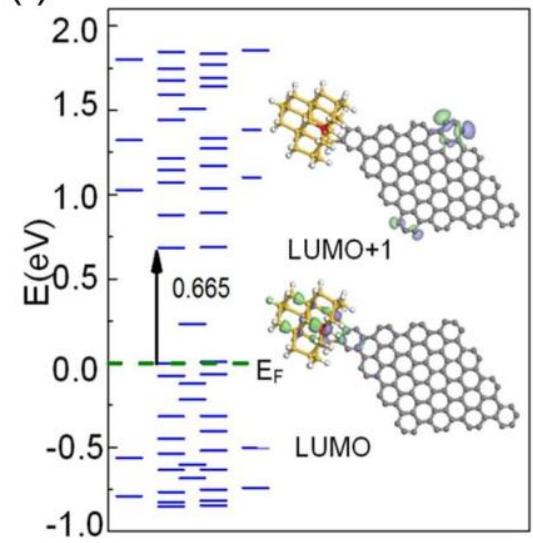

Figure S3. Ground-state electronic structures of (a) GN1, (b) GN2, (c) GN3, (d) SiB-GN1, (e) SiB-GN2, and (f) SiB-GN3. SiB nanoparticles are all terminated by $\mathrm{H}$. Each blue line represents an energy state. Dashed lines show the $E_{F}=0$. Isosurface in blue $(+)$ and green $(-)$ show the electron clouds of the frontier molecular orbitals. Isosurface in pink (+) and yellow (-) show the other degenerate orbital. ES (red rectangle) are generated above $\mathrm{E}_{\mathrm{F}}$. 
(a)

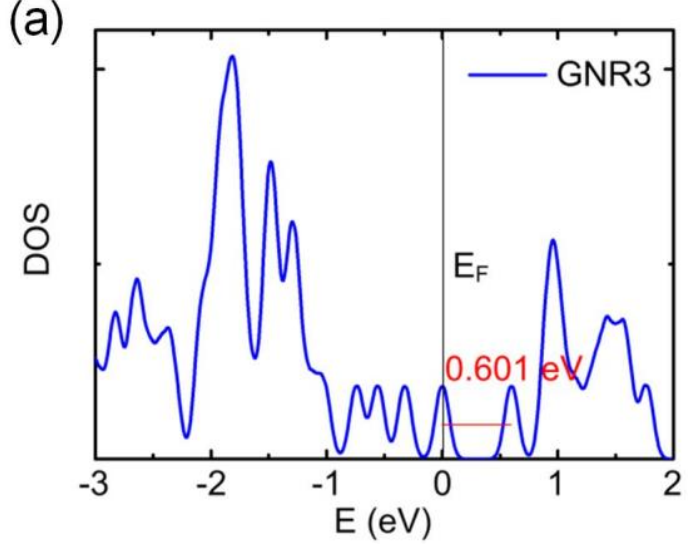

(b)

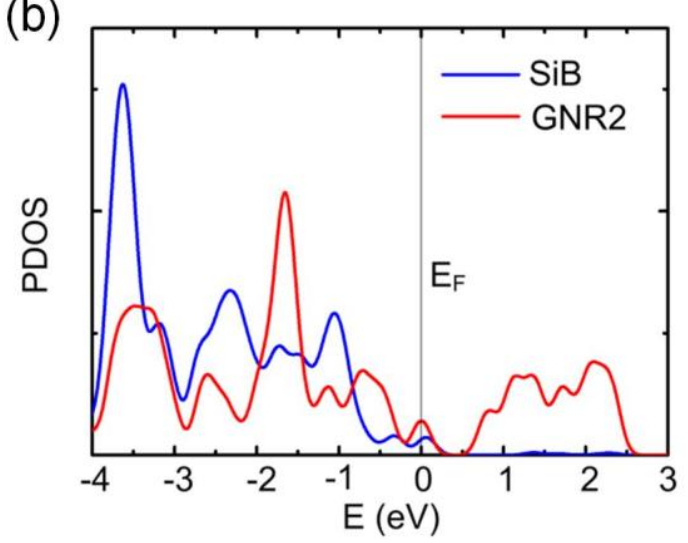

Figure S4. (a) Density of states (DOS) of GN3. Edge states open an energy gap of $0.601 \mathrm{eV}$, the smallest among GN1, GN2 and GN3. (b), Partial DOS (PDOS) of SiB-GN2. The resultant DOS of SiB-GN2 are decomposed into PDOS of SiB (blue) and of GN2 (red). SiB mainly contributes to the occupied states. The energy gap of SiB-GN2 is determined by GN2. 
Table S1. Distinct excited states and contributing MOs of SiB-GN1. HOMO of SiB-GN1 is $\psi(265-)$. The '+' and '-' denote spin up and spin down.

\begin{tabular}{ccccccccc}
\hline $\begin{array}{c}\text { Excited } \\
\text { States }\end{array}$ & From & & To & $\begin{array}{r}\text { TD-ex } \\
(\mathbf{e V})\end{array}$ & $\begin{array}{r}\text { KS-ex } \\
(\mathbf{e V})\end{array}$ & $\begin{array}{c}\text { TD-ex } \\
(\mathbf{n m})\end{array}$ & $\begin{array}{c}\text { KS-ex } \\
(\mathbf{n m})\end{array}$ & $\begin{array}{c}\text { Osc. } \\
\text { Stren. }\end{array}$ \\
\hline 1 & $258-$ & $\rightarrow$ & $271+$ & 1.35 & 1.04 & 916 & 1193 & 0.006184 \\
2 & $257-$ & $\rightarrow$ & $271+$ & 1.47 & 1.47 & 841 & 845 & 0.009782 \\
3 & $253-$ & $\rightarrow$ & $268+$ & 1.57 & 1.57 & 790 & 789 & 0.016785 \\
4 & $255-$ & $\rightarrow$ & $268+$ & 1.58 & 1.59 & 784 & 780 & 0.013668 \\
\hline
\end{tabular}
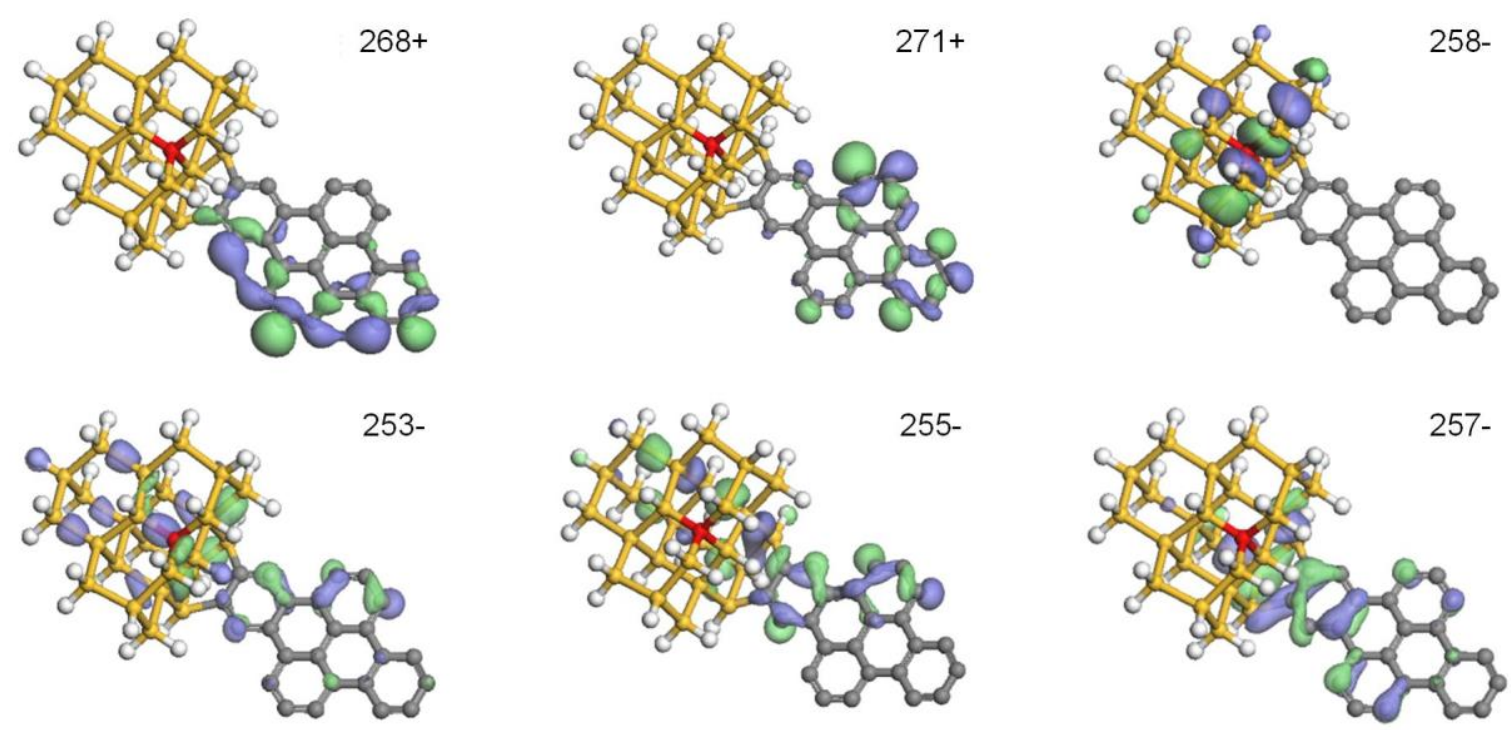

Figure S5. Contributing MOs of distinct excited state in SiB-GN1. Accepting MOs, $\psi(268+)$ and $\psi(271+)$ of SiB-GN1, both are edge states; Donating MOs, $\psi(253-)$, $\psi(255-), \psi(257-)$ and $\psi(258-)$ are $\mathrm{SiB} p$ electron bonding states, where GN $p$ electrons also join . 
Table S2. Distinct excited states and contributing MOs of SiB-GN2. HOMO of SiB-GN2 is $\psi(354-)$. The '+' and '-' denote spin up and spin down.

\begin{tabular}{ccccccccc}
\hline Excited & & & & TD-ex & KS-ex & TD-ex & KS-ex & Osc. \\
States & From & & To & $\begin{array}{c}\text { (eV) } \\
(\mathbf{e V})\end{array}$ & $(\mathbf{n m})$ & $\mathbf{( n m )}$ & Stren. \\
\hline 1 & $333-$ & $\rightarrow$ & $356+$ & 1.23 & 1.22 & 1007 & 1017 & 0.011945 \\
2 & $324-$ & $\rightarrow$ & $356+$ & 1.51 & 1.48 & 821 & 836 & 0.006072 \\
3 & $322-$ & $\rightarrow$ & $356+$ & 1.61 & 1.6 & 770 & 774 & 0.004556 \\
\hline
\end{tabular}
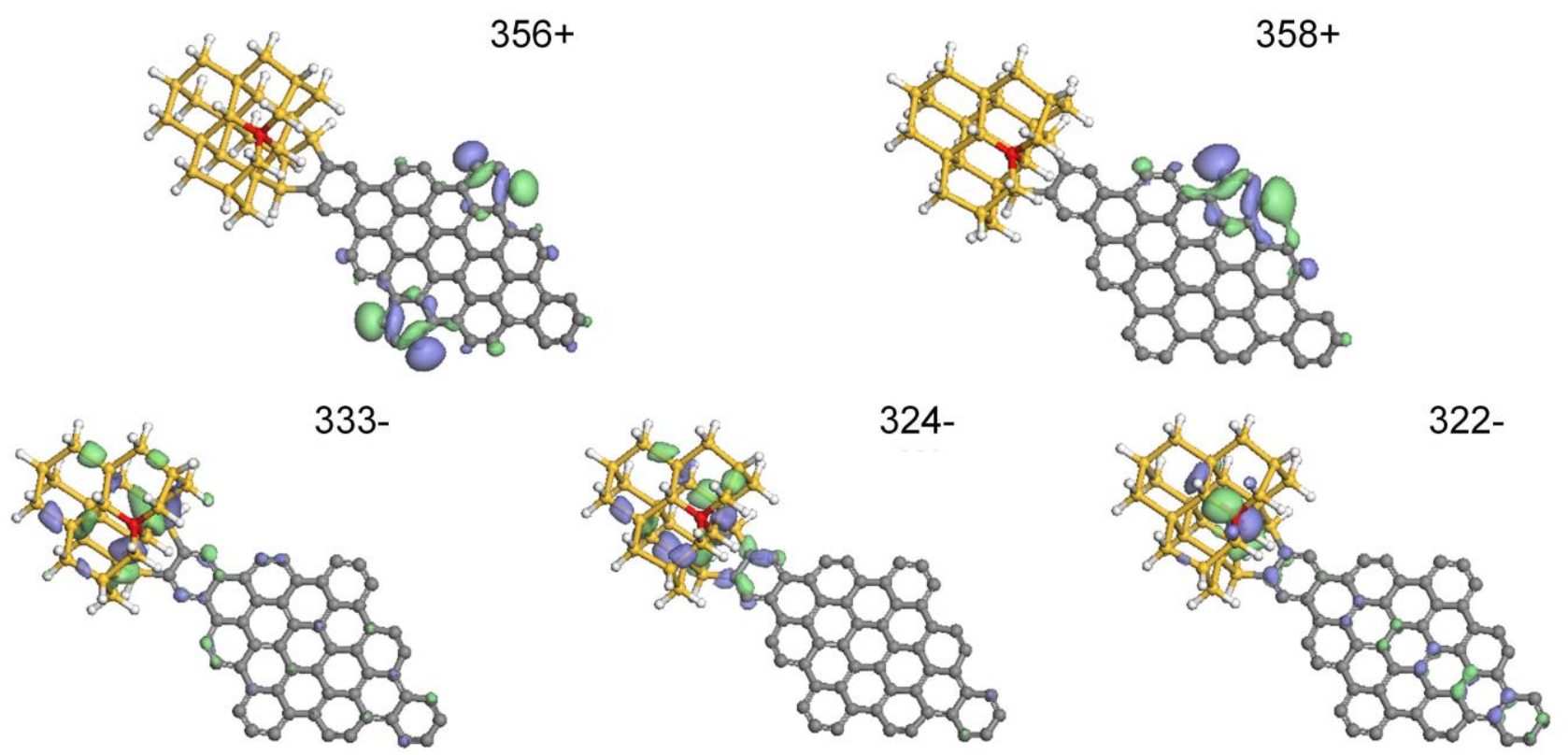

Figure S6. Contributing MOs of distinct excited state in SiB-GN2. Accepting MOs, $\psi(356+)$ and $\psi(358+)$ are edge states; Donating MOs, $\psi(333-), \psi(324-)$, and $\psi(322-)$ are $\mathrm{SiB} p$ electron bonding states, where GN $p$ electrons also join. 
Table S3. Distinct excited states and contributing MOs of SiB-GN3. HOMO of SiB-GN3 is $\psi(481-)$. The '+' and '-' denote spin up and spin down.

\begin{tabular}{ccccccccc}
\hline $\begin{array}{c}\text { Excited } \\
\text { States }\end{array}$ & From & & To & $\begin{array}{r}\text { TD-ex } \\
(\mathbf{e V})\end{array}$ & $\begin{array}{r}\text { KS-ex } \\
(\mathbf{e V})\end{array}$ & $\begin{array}{r}\text { TD-ex } \\
(\mathbf{n m})\end{array}$ & $\begin{array}{c}\text { KS-ex } \\
(\mathbf{n m})\end{array}$ & $\begin{array}{c}\text { Osci. } \\
\text { Stren. }\end{array}$ \\
\hline 1 & $469-$ & $\rightarrow$ & $482+$ & 0.69 & 0.7 & 1797 & 1784 & 0.036004 \\
2 & $467-$ & $\rightarrow$ & $482+$ & 0.78 & 0.78 & 1584 & 1584 & 0.008729 \\
3 & $467-$ & $\rightarrow$ & $482+$ & 0.78 & 0.79 & 1581 & 1562 & 0.135001 \\
4 & $465-$ & $\rightarrow$ & $482+$ & 0.86 & 0.87 & 1437 & 1431 & 0.008460 \\
\hline
\end{tabular}
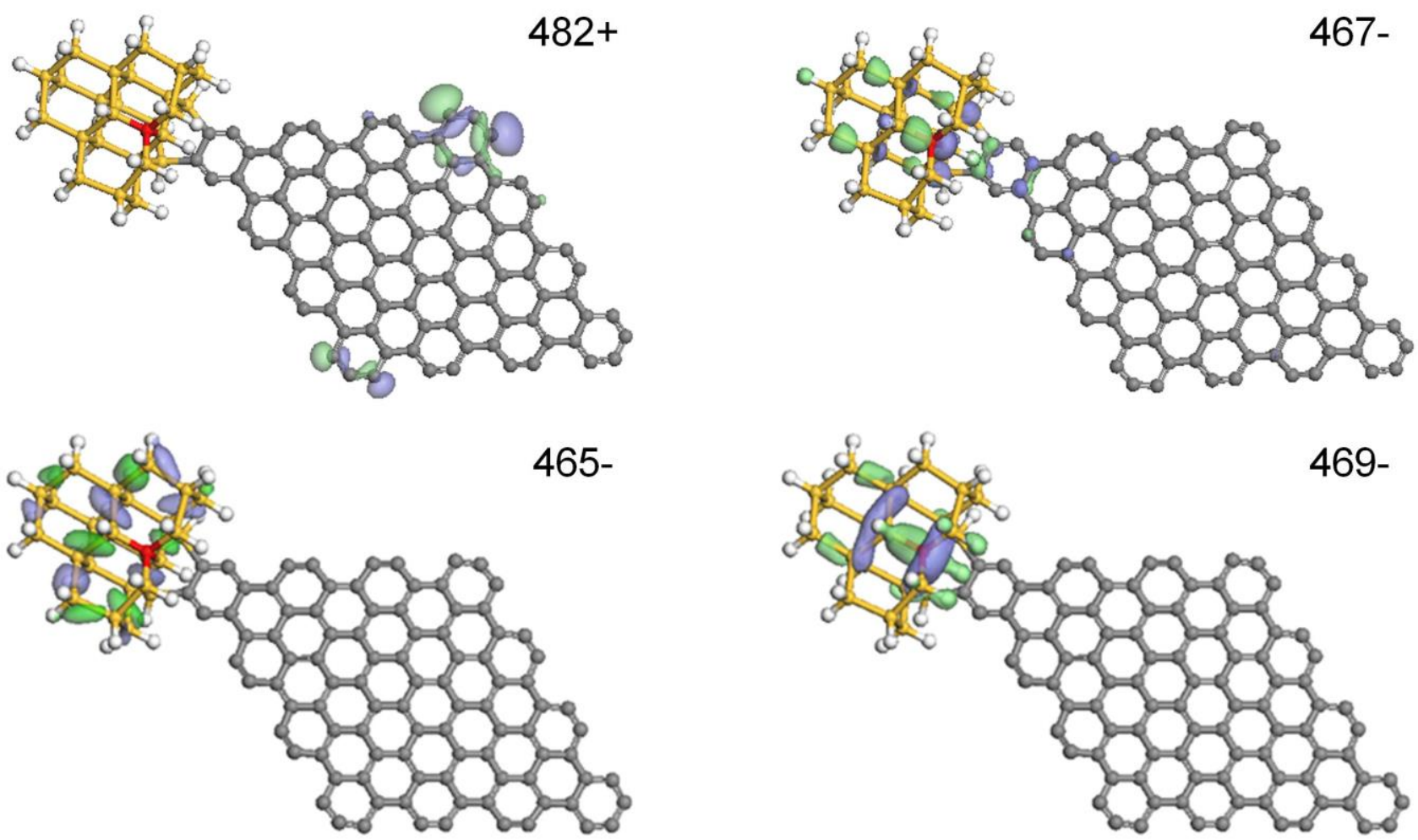

Figure S7. Contributing MOs of distinct excited state in SiB-GN3. Accepting MO, $\psi(482+)$ is edge state; Donating MOs, $\psi(469-), \psi(467-)$, and $\psi(465-)$ are $\mathrm{SiB} p$ electron bonding states. 


\section{References}

(1) Zhang, X.; Wang, C.; Sun, C. Q.; Diao, D. Magnetism induced by excess electrons trapped at diamagnetic edge-quantum well in multi-layer graphene. Appl. Phys. Lett. 2014, 105, 042402. 